\title{
Incidental Multifocal Peritoneal Adenomatoid Tumors Presenting Intra Operatively as Possible Metastatic Disease in a 73 Year Old Woman
}

\author{
Timlin DM, MBBchBAO, PhD ${ }^{1}$, Nolis T, MBBchBAO, MSc ${ }^{1}$, Treacy A, MBBchBAO, FRCPath ${ }^{1}$, \\ Thompson $C, M B B c h B A O^{2}$ and Mulligan NJ, MBBchBAO, ABPath
}

${ }^{1}$ Histopathology Department, Mater Misericordae University Hospital, Eccles Street, Dublin 7, Ireland

${ }^{2}$ Gynaecology Oncology Department, Mater Misericordae University Hospital, Eccles Street, Dublin 7, Ireland

\begin{abstract}
Adenomatoid tumour is a benign entity which occurs most commonly in the female and male genital tract. In females they cannot be distinguished clinically or radiologically from other entities such as leiomyoma; histology is required and intraoperative frozen section can be considered. Resection can be challenging when the growth pattern is diffuse. We report the finding of multiple peritoneal adenomatoid tumors discovered intra-operatively during a hysterectomy procedure for cervical squamous cell carcinoma (SCC) and ovarian fibromas. The multifocality raised concerns for a metastatic process. All visible deposits were successfully excised and histology confirmed multi-focal adenomatoid tumors. This case highlights a benign differential for an alarming intra-operative presentation, which could be important for guiding management and extent of surgical resection, especially where fertility is a concern.
\end{abstract}

\section{Background}

Adenomatoid tumors are benign, uncommon tumors of the female and male genital tracts composed of mesothelial cells. In females they are usually incidental findings in the uterus, ovary, fallopian tube, broad ligament and extra genital peritoneum. Extra pelvic presentations have been recorded; however these are exceedingly rare [1]. The usual age at presentation is during the reproductive years and at least one case is described associated with infertility, which resolved after surgery [2]. If detected radiologically, the pre-operative differential is leiomyoma, adenomyosis, endometriosis or malignancy. The differentials cannot be distinguished by clinical exam or imaging and histopathology is required. The treatment of choice is complete surgical clearance although this can be difficult as the tumour arises from and, is surgically indistinct from the mesothelium of the serosa.

Histologically, many patterns are described, including adenoid, angiomatoid, cystic, tubular, oncocytic and solid [3]. They have the unifying feature of bland cytology, often surrounded by but not encapsulated with, normal mesothelial cells. They have been shown to be of mesothelial origin and this is reflected in the immunohistochemical (IHC) staining pattern

\section{Case Presentation}

Our patient is a 73-year-old woman who presented with abdominal pain and what was felt to be a rapidly expanding uterine fibroid. Cervical biopsy at the time of a hysteroscopy revealed high grade $\mathrm{CIN}$ which was treated by large loop excision of transformation zone (Lletz). Endometrial currettings revealed high grade cervical intraepithelial neoplasia (CIN) which was treated by Lletz. Radiology revealed limited disease, however it was noted that bilateral ovarian fibromas had increased in size to $11 \mathrm{~cm}$ and $15 \mathrm{~cm}$ but no other distant disease. Tumour markers were within normal range. Lletz analysis revealed micro invasive SCC with $<1 \mathrm{~mm}$ depth (FIGO Stage 1A1). In light of this, surgery was progressed following MDT discussion. Intra-operatively, bilateral solid ovarian masses were found alongside multiple small nodules (3-11 $\mathrm{mm}$ ) on the broad ligament, fallopian tube (Figure 1), bladder peritoneum, sigmoid epiploica and small bowel mesentery, which were concerning for malignancy. A frozen

*Corresponding author: Deirdre Timlin, MbBChBAO, PhD, Department of Histopathology, Mater Misericordiae University Hospital, Dublin 7, Ireland, Tel: 00353-87-6112166

Accepted: January 29, 2022

Published online: January 31, 2022

Citation: Timlin DM, Nolis T, Treacy A, et al (2022) Incidental Multifocal Peritoneal Adenomatoid Tumors Presenting Intra Operatively as Possible Metastatic Disease in a 73 Year Old Woman. J Clin Surg Pathol 4(1):34-37 
Citation: Timlin DM, Nolis T, Treacy A, et al (2022) Incidental Multifocal Peritoneal Adenomatoid Tumors Presenting Intra Operatively as Possible Metastatic Disease in a 73 Year Old Woman. J Clin Surg Pathol 4(1):34-37

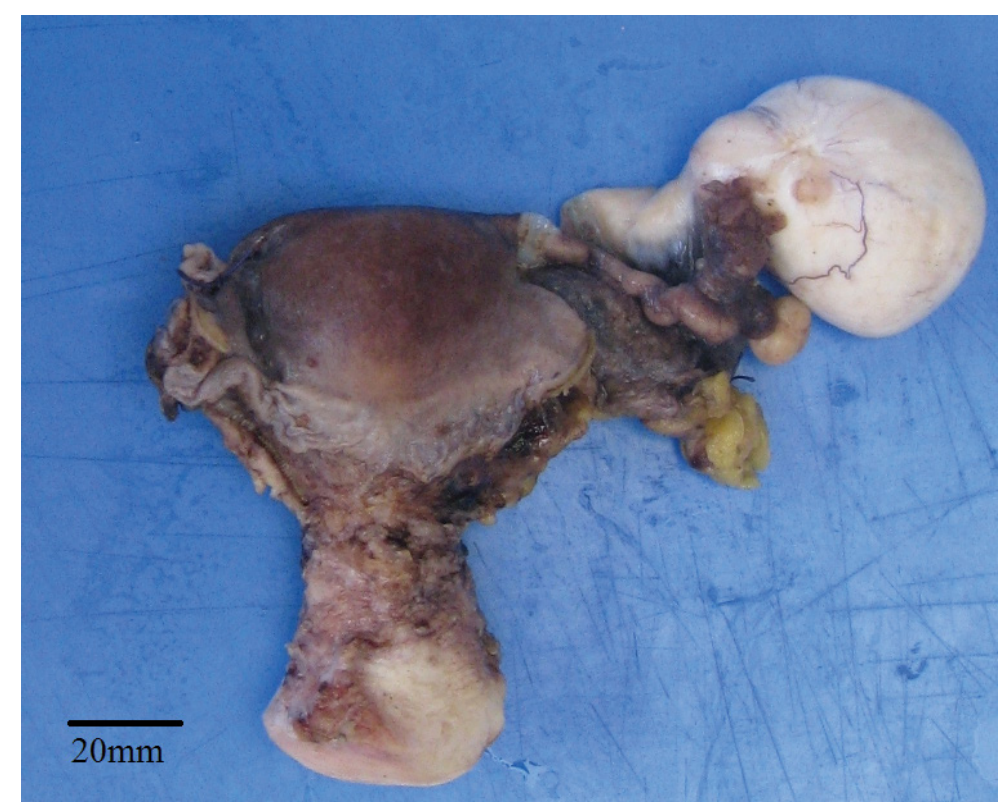

Figure 1: Gross specimen, uterus, left fallopian tube and ovary with fibroma. Lesions circled on the surface of the fibroma and in continuity with broad ligament near the fallopian tube were sampled.

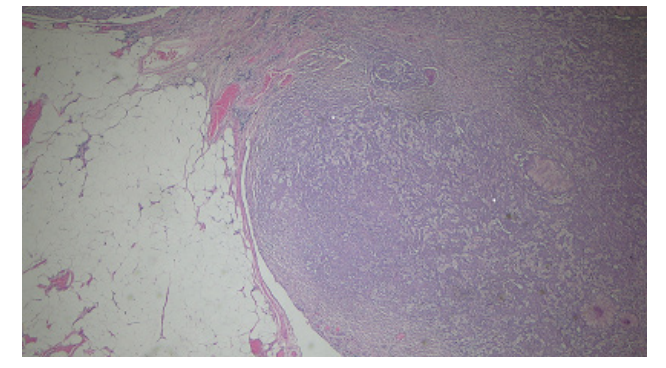

A

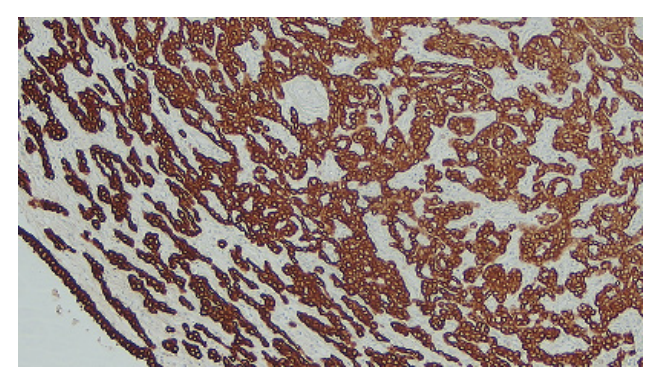

C

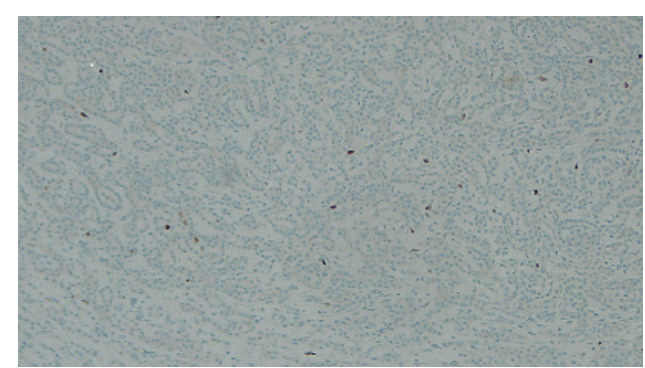

E

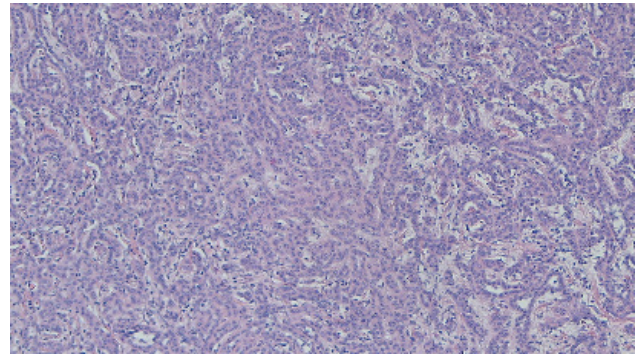

B

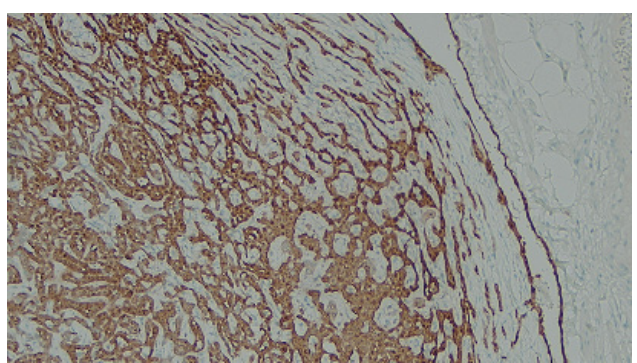

D with bland cytology (100x HE); (c) Cam5.2; (d) CALR; (e) ki67. 
Citation: Timlin DM, Nolis T, Treacy A, et al (2022) Incidental Multifocal Peritoneal Adenomatoid Tumors Presenting Intra Operatively as Possible Metastatic Disease in a 73 Year Old Woman. J Clin Surg Pathol 4(1):34-37

section was considered but ruled out based on duration of that procedure, patient age and accessability achieving full resection. Therefore, a total abdominal hysterectomy with bilateral salpingo-oophrectomy, omentectomy, lymph node sampling and excision of all peritoneal nodules was carried out and sent for histology. Complete clearance was achieved.

Pathology revealed residual cervical CIN 2-3 with clear margins and no invasive disease, a $10 \mathrm{~mm}$ intramural leiomyoma in the uterus and bilateral ovarian fibromas. These were extensively sampled and they were found to be pure fibromas with no evidence of sex cord elements. Lymph nodes were reactive and negative for malignancy. The nodules seen at surgery were tumors composed of anastomosing cords, tubules and papillary structures, arising in association with the mesothelial layer of the broad ligament specimen (Figure 2a). Cytologically the tumors were bland with regular, central nuclei, even chromatin and moderate cytoplasm. Mitoses were rare and there was no necrosis. Lymphoid follicles were present in nodules from the sigmoid epiploica and small bowel mesentery.

IHC was used to rule out metastatic disease from both her known early stage cervical cancer and to outrule a possible occult malignancy. Mesothelial markers CALR, D240, WT-1 and EMA were positive, as was Pax-8, AE1/3, Cam5.2 and CK7, with weak CK5/6 staining. No staining was seen with p63, GATA3, CK20, CD10 or neuroendocrine markers (Figure 2). The ki67 proliferation rate was $5 \%$. A well differentiated malignant mesothelioma was also a diagnostic consideration, however this was considered unlikely given the bland cytology and architecture and extremely rare mitoses. Mesothelioma can be more confidently ruled out in suspicious cases ruled out on the basis of positive BAP-1 staining, however this stain was not available to us at this time. Based on morphology and the reported stains a diagnosis of multifocal adenomatoid tumors was reached.

\section{Conclusions}

Adenomatoid tumors were first described by Golden and Ash in 1945 [4]. Immunohistochemical and ultrastructual studies since then have shown them to be derived from mesothelial cells. Their predilection for the genital tract remains unsolved. The behaviour described thus far is benign, however they remain a therapeutic challenge as they cannot be distinguished from their radiologic differentials of leiomyoma, adenomyosis or endometriosis without histology [3]. Given their mesothelial origins, these tumors mark with mesothelial antibodies. Our mesothelial markers WT-1, CALR, D-240 and EMA were positive whilst Ck5/6 was weak and BerEp4 was negative. We found reactivity against the broad spectrum cytokeratins $A e 1 / 3$ and Cam5.2. In a series of 44 adenomatoid tumors of both the male and female genital tracts Sangoi [5] describe similar IHC profiles with CALR, WT-1 and pancytokeratin positivity.

Metastatic SCC was ruled out with negative p63 staining, other ovarian and uterine mesenchymal tumors were ruled out by negative inhibin and CD10 staining. Neurendocrine markers synaptophysin and chromogranin were also negative.
We describe a rare entity presenting in an unusual way, raising concerns for metastatic disease. Intra-operative assessment of these lesions is fraught with difficulty as the only definitive test is histopathology. Removal of the lesions themselves is technically challenging due to their merging with normal mesothelium, offering no clear plane of resection. The inability to achieve complete clearance is most likely the reason behind the 'recurrence' of a single case in 2005 [2]. In the current case, we describe discrete nodules occurring at multiple sites including the small bowel mesentery and presumed to be related to the largest nodule found on the broad ligament/ fallopian tube. Extra pelvic adenomatoid tumors have been described and given their mesothelial origin it is unsurprising that pleural and mediastinal cases exist, however this entity is also described in the adrenals and liver where it is assumed to derive from mesothelial rests [610].

Adenomatoid tumors may occur as single or uncommonly, as multifocal masses. Most of the cases described with multifocal disease were associated with some form of immunosuppression. The first, described in 1992, was of a woman who had undergone kidney transplantation for complications associated with Lupus. She was found to have an adenomatoid tumour diffusely infiltrating the entire myometrium, with a focally papillary cystic architecture [10]. A second case in 2000 [11] and another in 2003 [12] occurred in patients with renal failure and multifocal adenomatoid tumors. Other cases have been described in, usually elderly, patients undergoing investigation for a concurrent disease [13]. The link, if any, between immunosuppression and the genesis of this tumour is unclear. Whilst our patient was not immunosuppressed due to an underlying illness, given her advanced age, it is not unreasonable to suggest that this might impact the natural history of untreated adenomatoid tumors.

This case shows that diffuse/multifocal adenomatoid tumors should be considered in the differential diagnosis when faced with apparent widespread metastatic peritoneal disease. Frozen section could be considered as a diagnostic aid to help guide tissue excision limits in cases of reproductiveage women.

\section{References}

1. Oliviera GF (2015) Adenomatoid tumour of myometrium: a case report and review of the literature. Dissertation: Dissertação para obtenção do Grau de Mestre em Medicina (Ciclo de estudos integrado).

2. Sieunarine A, Cowie AS, Bartlett JD, et al. (2005) A novel approach in the management of a recurrent adenomatoid tumour of the uterus utilising a Strassmann technique. Int J Gynecol Cancer 15: 671-675.

3. Sangoi AR, McKenney JK, Schwartz EJ, et al. (2009) Adenomatoid tumours of the female and male genital tracts: a clinicopathological and immunohistochemical study of 44 cases. Mod Pathol 22: 1228-1235.

4. Golden A, Ash JE (1945) Adenomatoid tumours of the genital tract. Am J Pathol 11: 288-292.

5. Parekh V, Winokur T, Cerfolio RJ, et al. (2016) Posterior 
mediastinal adenomatoid tumour: A case report and review of the literature. Case Rep Pathol 2016: 1-5.

6. Malpica A, Euscher ED, Marques-Piubelli ML, et al. (2021) Malignant Mesothelioma of the Peritoneum in Women: A Clinicopathologic Study of 164 Cases. Am J Surg Pathol 45: 45-58.

7. Nagata S, Aishima S, Fukuzawa K, et al. (2008) Adenomatoid tumour of the liver. J Clin Pathol 61: 777-780.

8. Natarajan S, Luthringer DJ, Fishbein MC (1997) Adenomatoid tumor of the heart: report of a case. Am J Surg Pathol 21: 13781380.

9. Kaplan MA, Tazelaar HD, Hayashi T, et al. (1996) Adenomatoid tumors of the pleura. Am J Surg Pathol 20: 1219-1223.
10. Livingston EG, Guis MS, Pearl ML, et al. (1992) Diffuse adenomatoid tumor of the uterus with a serosal papillary cystic component. Int J Gynecol Pathol 11: 288-292.

11. Tiras MB, Noyan B, Suer O, et al. (2000) Adenoamtoid tumour of the uterus in a patient with chronic renal failure. European Journal of Obstetrics and Gynecoligcal Reproductive Biology 92: 205-207.

12. Cheng CA, Wee A (2003) Diffuse uterine adenomatoid tumour in an immunosuppressed renal transplant recipient. Int J Gynaecol Pathol 22: 198-210.

13. Hayes SJ, Clark P, Mathias R, et al. (2007) Multiple adenomatoid tumours in the liver and peritoneum. J Clin Pathol 60: 722-725. 